means of a transformation of Lie, Dr. Gundelfinger effects a classification of ordinary differential equations of the first order with respect to the arrangement of the $\infty^{2}$ osculating circles to their integral curves and develops a theory of reciprocal line element loci.

F. N. CoLe, Secretary.

\title{
THE APRIL MEETING OF THE CHICAGO SECTION.
}

The twenty-seventh regular meeting of the Chicago Section of the American Mathematical Society was held at the University of Chicago on Friday and Saturday, April 8-9, 1910. Professor L. E. Dickson, Vice-President of the Society and Chairman of the Section, presided at the three sessions held on Friday morning and afternoon and Saturday morning. The attendance at the various sessions included twenty visitors and the following forty-four members of the Society:

Mr. R. P. Baker, Mr. W. H. Bates, Professor G. A. Bliss, Professor Oskar Bolza, Dr. R. L. Börger, Dr. H. E. Buchanan, Dr. Thomas Buck, Dr. H. T. Burgess, Dr. A. R. Crathorne, Professor D. R. Curtiss, Professor L. E. Dickson, Dr. Arnold Dresden, Professor W. B. Ford, Professor A. G. Hall, Professor E. R. Hedrick, Mr. T. H. Hildebrandt, Professor O. D. Kellogg, Professor Kurt Laves, Dr. A. C. Lunn, Dr. W. D. MacMillan, Mr. E. J. Miles, Professor G. A. Miller, Dr. R. L. Moore, Professor E. H. Moore, Professor J. C. Morehead, Professor F. R. Moulton, Dr. L. I. Neikirk, Dr. Anna J. Pell, Professor Alexander Pell, Professor H. L. Rietz, Miss Ida M. Schottenfels, Mr. A. R. Schweitzer, Professor J. B. Shaw, Mr. R. R. Shumway, Professor C. H. Sisam, Professor H. E. Slaught, Professor E. J. Townsend, Professor A. L. Underhill, Professor E. B. Van Vleck, Professor E. J. Wilczynski, Professor B. F. Yanney, Professor J. W. Young, Professor J. W. A. Young, Professor Alexander Ziwet.

On Friday evening nearly all of the members present at the meeting dined together at the Quadrangle Club, at which time the question of holding the next meeting of the Section at Minneapolis was discussed. A letter was read from the President of the University of Minnesota urging the Section to meet 
there in connection with the annual convocation of the American Association for the Advancement of Science. Both the president and secretary of Section A of the Association, Professors E. H. Moore and G. A. Miller, urged the desirability of closer affiliation between the mathematicians and other scientists, especially the astronomers and physicists. It was finally voted to hold the December meeting of the Section at Minneapolis, unless the Council should decide that the annual meeting of the Society, with the presidential address, will be held at Chicago at that time. It had been previously announced that the Council had considered favorably the question of holding a meeting of the entire Society at Chicago either in December, 1910, or in April, 1911, depending upon the convenience of the President, Professor Bôcher.

The following papers were read at this meeting:

(1) Professor D. R. Curtiss: "An extension of Descartes's rule of signs."

(2) Professor C. H. Sisam : "On a class of $r$-spreads in space of $n$ dimensions."

(3) Dr. Anna J. Pell : "On a functional equation."

(4) Dr. H. E. Buchanan : "The equations of variation for the straight line elliptical orbits of three finite masses."

(5) Professor J. B. Shaw : "On quaternions."

(6) Professor W. B. Fond : "On the determination of the asymptotic developments of a given function (second paper)."

(7) Professor Oskar Bolza : "An application of 'general analysis' to a problem in the calculus of variations."

(8) Dr. H. T. BuRGESS : "Differential equations of the projective curves on a quadric whose tangents belong to a line complex."

(9) Professor E. J. Wilczynski : "On the general theory of congruences of straight lines."

(10) Mr. R. P. BAKER : "On a class of equations of state representing normal and abnormal three-state bodies."

(11) Dr. L. I. NeIKIRK : "A theorem on $(m, n)$ correspondence."

(12) Mr. W. H. Bates: "Note on the generalization of the formulas of Gauss and Codazzi."

(13) Mr. W. H. Bates: "On the medium curvature of a hypersurface (second paper)."

(14) Professor G. A. Mrller : "Groups of transformations of Sylow subgroups." 
(15) Professor G. A. Mrller : "Extensions of theorems due to Cauchy."

(16) Dr. R. L. Moore : "On Duhamel's theorems."

(17) Miss Ida M. SchotTenfels: "The Fano geometry."

(18) Professor F. R. Moulton: "On the problem of the spherical pendulum."

(19) Professor L. E. Drckson : "Equivalence of families of quadratic forms."

Professor Dickson's paper was read by title. Professor Bolza's paper appeared in full in the May number of the Bulletrin. Abstracts of the other papers follow below, the numbers corresponding to those in the above list.

1. Descartes's rule of signs gives merely an upper limit for the number of positive roots of an algebraic equation $f(x)=0$. To determine the exact number of such roots we must usually have recourse to other methods. In Professor Curtiss's paper it is, however, shown that there always exists a polynomial $\phi(x)$ such that the number of variations of sign in the coefficients of $F(x)=f(x) \phi(x)$ is exactly equal to the number of positive roots of $f(x)=0$. Further, $\phi(x)$ can be so chosen that $F(x)$ has only $m+1$ coefficients, where $m$ is the degree of $f(x)$, and these coefficients are determinants of a matrix formed from the coefficients of $f(x)$. From this is derived a criterion for the number of real roots in any interval $(a, b)$. The paper concludes with a graphical method for applying these tests.

2. The principal theorem in Professor Sisam's paper is the following: If $V_{r}$ is an analytic $r$-spread belonging to a space of $n$ dimensions $(n \geqq r+1)$; if through a generic point of $V_{r}$ there does not pass a continuum of spaces of $t$ dimensions lying on $V_{r}$; and if the section of $V_{r}$ by a generic hyperplane through the tangent $r$-dimensional space at a generic point $P$ of $V_{r}$ is an $(r-1)$-spread the tangent cone to which at $P$ has a double $t$-dimensional space; then $V_{r}$ is generated by $t$-dimensional spaces in such a way that the tangent $r$-dimensional space to $V_{r}$ is invariant along each $t$-dimensional space.

3. The functional equation $\lambda \phi(s)=T[\phi(s)]$, where $T$ is a single-valued fractional transformation with the two properties

$$
\int_{a}^{b} f(s) T[f(s)] d s \geqq 0,
$$

$\left(f_{1}, f_{2}\right) \quad \int_{a}^{b} f_{1}(s) T\left[f_{2}(s)\right] d s=\int_{a}^{b} f_{2}(s) T\left[f_{1}(s)\right] d s$, 
is reduced by means of a biorthogonal system of functions to an infinite system of linear equations whose coefficients form a limited symmetric matrix. Dr. Pell considers the character of solutions corresponding to a point, or continuous spectrum of $\lambda$, and finds conditions for the solvability of the non-homogeneous functional equation, together with some expansion theorems.

4. In his dissertation Dr. Buchanan discussed some periodic orbits of three bodies near the straight line circular solutions. The present paper is a report on the extension to the straight line elliptical solutions. For $e=0$, the solutions obtained here reduce to those obtained in the circular case. The equations of variation, formed in the usual way, are a system of homogeneous linear equations of the twelfth order, but two of them are independent of the other four. The coefficients of the equations are expanded as power series in the eccentricity, the coefficients of these power series being simply periodic. The solutions are found to exist as power series in $e$. The characteristic exponents are found to be uniquely determined by the periodicity conditions and to have the same character as in the circular case.

5. In this paper Professor Shaw develops formulas for certain functions of two, three, four, or more quaternions, by means of which the various differentiating operators of quaternions may be readily expressed. Thus formulas are found for the quaternion operator

$$
\frac{\partial}{\partial w}+i \frac{\partial}{\partial x}+j \frac{\partial}{\partial y}+k \frac{\partial}{\partial z}
$$

which are as manageable as those for $\nabla$. Theorems of integration are given. These functions furnish an application to quaternions of general formulas previously given in the author's papers on general associative algebra.

6. In Professor Ford's paper a method is derived for determining the asymptotic developments of a given function, and application is made to a number of important functional types. The results supplement those of a former paper (see April number of the Annals of Mathematics); also those of Barnes, Lindelöf, Wiman, Mattson, and others concerning the asymptotic develments of integral functions defined by canonical (Weierstrass) 
products. A new type of development is obtained for these latter functions, thus enabling one to study their behavior for large values of the variable in regions which were precluded in the previous developments.

8. Dr. Burgess shows that the differential equation of the curves, upon the quadric $\phi(x) \equiv x_{1} x_{4}+x_{2} x_{3}=0$, whose tangents belong to the line complex $F\left(p_{12}, p_{13}, p_{14}, p_{34}, p_{42}, p_{23}\right)=0$, is homogeneous in six arguments and of the form

$$
F\left(u^{2} d v, v^{2} d u,-u d v-v d u,-d v, d u, v d u-u d v\right)=0,
$$

where the parametric equations of the quadric $\phi$ are

$$
\rho x_{1}=u v, \quad \rho x_{2}=u, \quad \rho x_{3}=v, \quad \rho x_{4}=-1 .
$$

The case for a linear line complex is then studied in detail and the theory of elementary divisors is applied to get the canonical forms of the differential equations, together with their integrals. Next, the case where $F(p)=0$ is quadratic is attacked in the particular form in which $F(p)=0$ is the line equation of a quadric surface. Here the theory of elementary divisors is again applied and the canonical forms of the differential equations are found.

9. The new theory of congruences presented by Professor Wilczynski is based upon the consideration of the completely integrable system of partial differential equations

$$
\begin{aligned}
& y_{v}=m z, \quad z_{u}=n y, \\
& y_{u u}=a y+b z+c y_{u}+d z_{v}, \\
& z_{v v}=a^{\prime} y+b^{\prime} z+c^{\prime} y_{u}+d^{\prime} z_{v} .
\end{aligned}
$$

The integral surfaces of $(D)$ are the two sheets of the focal surface of the congruence, the curves $u=$ const. and $v=$ const. upon these surfaces being their intersections with the developables of the congruence. The lines of the congruence are obtained by joining corresponding points $(u, v)$ of the two surfaces. All of the known results in the general theory of congruences, so far as they are of a projective nature, present themselves in a very simple way from this point of view, and a large number of new theorems make their appearance. The so-called Laplace transformation which was first associated with the theory of congruences by Darboux appears in a new light, enabling one 
to formulate and solve far more general problems than has hitherto been possible. The general theory is applied to two particular problems : first, to the determination of those congruences which belong to a linear complex and for which the first Laplace transformation gives rise to a congruence that likewise belongs to a linear complex; second, to the study of those congruences whose focal surfaces are quadrics. Both of these problems are closely connected with the differential equation

$$
\frac{\partial^{2} \theta}{\partial u \partial v}=e^{2 \theta}-e^{-2 \theta}
$$

10. The equations considered in Mr. Baker's paper are of the form $p=t F-T M$, in which $F$ represents the gas law and is explicitly discussed for Van der Waal's form $1 /(v-1)$ and for Planck's form - log $(1-1 / v) ; M=1 / v^{2}-c / v^{3}+d / v^{4}$ and is an extension of Van der Waal's term which may ultimately be a power series; and $T=m t /\left(t^{2}+k t+l^{2}\right)$ is a special form of Amagat's multiplier. The conditions for the existence of a triple point when $T=1$ are worked out for both the gas laws mentioned and give in each case an area in the $(c, d)$ plane within which triple points are possible. If $k>0$, the surface is of the same type as for a normal three state body, the $L S$ line ending at a critical point or not, according to the dependence of $c, d$ on the temperature, quite small movements of the $(c, d)$ point deciding. If $k<0$ and the $(c, d)$ point passes out of the triple point area before the critical temperature is reached, the equation represents the type of an abnormal body. If this does not happen, a form approximating the "Tamann idea" is reached, though the complete form requires seven values of $v$ for given $p, t$. The particular form of the gas law is indifferent as far as type is concerned; and the reversal of sign of the molecular pressure is neither necessary nor sufficient for abnormality, but may occur with a very low temperature for the triple point. By a proper choice of the three scales and the constants, the equations may be compared with the observations.

11. Emil Weyr in a paper * published in 1870 defines an $n$ fold involution of points on a straight line as the points whose abscissas are roots of the equation $f(x)-\lambda \phi(x)=0$, where the base functions $f(x)$ and $\phi(x)$ are rational integral functions of

* Math. Annalen, vol. 3 (1870), pp. 34-44. 
degree $n$, and $\lambda$ is an arbitrary parameter. He finds its double elements and other properties. In a second paper $\dagger$ he introduces the two involutions $f(x)-\lambda \phi(x)=0$ and $F(y)-\mu \psi(y)=0$, of degrees $m$ and $n$, and calls these involutions projective when $A \lambda \mu+B \lambda+C \mu+D=0$. He considers the plane curves defined by the projective involutions and develops their properties. Dr. Neikirk considers an $(m, n)$ correspondence given by a rational integral equation $f(x, y)=0$ of degree $m$ in $x$ and $n$ in $y$ which has at least a single set of correspondences of the type $\left(x_{1}, x_{2}, x_{3} \ldots, x_{m} ; y_{1}, y_{2}, y_{3} \ldots, y_{n}\right)$ and shows that $f(x, y)=0$ may be reduced to $\theta(x)+\lambda \phi(x)=0, \chi(y)+\lambda \psi(y)=0$, where $\theta, \phi, \chi$, and $\psi$ are rational integral functions, the first two of degree $m$, the last two of degree $n$, and $\lambda$ is a variable parameter.

12. In his first paper Mr. Bates uses Maschke's symbolic notation in generalizing the ordinary method of deriving the Gauss and Codazzi formulas.

13. In a paper read before the Society in December, 1909 , Mr. Bates expressed those medium curvatures of $R_{n}$ that have even subscripts, in terms of the first fundamental quantities of $R_{n}$. In the present paper, he makes use of a theorem of Maschke to express those medium curvatures of $R_{n-1}$ that have odd subscripts, in terms of the first fundamental quantities of $R_{n}$ and the function which defines $R_{n-1}$ in $R_{n}$. The formula for the first medium curvature $K_{1}$ contains as special cases the curvature of ordinary plane and twisted curves and the medium curvature of the surface $f(x, y, z)=0$.

14. Professor Miller proves the following theorems: The necessary and sufficient condition that a transitive group of degree $n$ is a group of transformations of Sylow subgroups is that its subgroup composed of all its substitutions which omit a given letter involves one and only one Sylow subgroup of degree $n-1$. Hence there are only two alternating and also only two symmetric groups which are groups of transformations of Sylow subgroups. If the Sylow subgroups of order $p^{m}$ in a group are transformed under this group according to an imprimitive group, the number of these Sylow subgroups is of the form $\left(1+k_{1} p^{a}\right)\left(1+k_{2} p^{a}\right)$ where none of the integers $k_{1}, k_{2}, \alpha$ is zero. Moreover, the systems of imprimitivity must be transformed

$\dagger$ Prager Sitzungsberichte, 1870, pp. 14-19. 
according to some non-regular group. If a group involves only 3 Sylow subgroups of order $2^{m}$ these subgroups generate a group of order $3.2^{m}$; if it involves exactly 4 Sylow subgroups of order $3^{m}$ they must generate a group whose order is either $4.3^{m}$ or $8.3^{m}$; if it involves exactly 6 Sylow subgroups of order $5^{m}$ they generate a group whose order is either $12.5^{m}$ or $24.5^{m}$. The group generated by these Sylow subgroups is a characteristic subgroup of the entire group if it does not coincide with it.

15. The object of Professor Miller's second paper was to extend a theorem due to Cauchy and to indicate how Sylow's theorem is an almost immediate result of this extended theorem, which was stated as follows: The number of those operators of a group $G$, involving $H_{1}, H_{2}$ as subgroups, which transforms $H_{1}$ into a group having exactly $\rho$ operators in common with $\mathrm{H}_{2}$ is divisible by $h_{1} h_{2} / \rho, h_{1}$ and $h_{2}$ being the orders of $H_{1}$ and $H_{2}$ respectively. This theorem was proved by Cauchy for the special case where $\rho=1$, and this special case was used by him to prove that every group whose order is divisible by a given prime number $p$ must involve operators of order $p$. The extended theorem may be used, in a similar way, to prove that every group whose order is divisible by $p^{a}$ must involve a subgroup of order $p^{a}$.

16. Dr. Moore discusses certain conditions under which the limit of the sum of one set of infinitesimals is the same as that of another set.

17. In the Annals of Mathematics, second series, volume 11, number 2, page 60, Mr. George M. Conwell discusses the Fano configuration and its group. Miss Schottenfels in the present paper discusses the postulates under which the Fano geometry was developed and its extension to $n$-space. See Giornali di Matematiche, volume 30 (1892).

18. The problem of the spherical pendulum gives rise to the differential equations

$$
\begin{gathered}
\frac{d^{2} x}{d t^{2}}+\left(a_{0}+a_{1} z\right) x=0, \frac{d^{2} y}{d t^{2}}+\left(a_{0}+a_{1} z\right) y=0, \\
\frac{d^{2} z}{d t^{2}}+\left(b_{0}+b_{2} z^{2}\right) z-g=0,
\end{gathered}
$$

where $x, y$ and $z$ are subject to the conditions

$$
x^{2}+y^{2}+z^{2}=l^{2} .
$$


Professor Moulton treats the third equation as one of a class possessing periodic solutions. The existence of the solutions is proved and practical methods of constructing them as power series in a parameter are given. Then the first two equations become homogeneous linear differential equations having simply periodic coefficients of the form first treated by Hill in his memoir on the motion of the lunar perigee. A distinct treatment of these equations is given, both as to the existence of the solutions and as to practical methods of finding them.

19. Professor Dickson discusses the equivalence of two pencils of quadratic forms in $n$ variables. If in the pencil $A=\lambda_{1} A_{1}+\lambda_{2} A_{2}$, we replace the generators $A_{1}$ and $A_{2}$ by $A_{1}=g A_{1}+h A_{2}$ and $\bar{A}_{2}=g^{\prime} A_{1}+h^{\prime} A_{2}$, where $g h^{\prime}-g^{\prime} h \neq 0$, then $\bar{A}=\lambda_{1} \bar{A}_{1}+\lambda_{2} \bar{A}_{2}$ becomes $\bar{\lambda}_{1} A_{1}+\bar{\lambda}_{2} A_{2}$, where $\bar{\lambda}_{1}=\lambda_{1} g+\lambda_{2} g^{\prime}, \bar{\lambda}_{2}=\lambda_{1} h+\lambda_{2} h^{\prime} . \quad$ An elementary divisor $\left(a_{1} \lambda_{1}+a_{2} \lambda_{2}\right)^{a}$ of $|A|$ corresponds to the elementary divisor $\left(\bar{a}_{1} \lambda_{1}+\bar{a}_{2} \lambda_{2}\right)^{a}$ of $|\bar{A}|$, where $\bar{a}_{1}=a_{1} g+a_{2} h, \bar{a}_{2}=a_{1} g^{\prime}+a_{2} h^{\prime}$. It follows that two non-singular pencils of quadratic forms are equivalent if and only if the elementary divisors of one pencil can be derived from those of the second by a linear transformation on the two parameters.

To discuss the problem of equivalence in a given field $F$, use is made of the results in Transactions, volume 10 (1909), pages $347-360$. For the above replacement of generators, the elementary divisor $(\lambda-c)^{a}$ of $\left|\lambda A_{1}-A_{2}\right|$ corresponds to the elementary divisor $(\lambda-\gamma)^{a}$ of $\left|\lambda \bar{A}_{1}-\bar{A}_{2}\right|$, where $\gamma=\left(g^{\prime}+c h^{\prime}\right) /(g+c h)$. Hence from the ultimate canonical types (obtained in the paper cited) of non-singular pairs of quadratic forms we may derive canonical types of pencils of forms by restricting the roots $c_{1}, c_{2}, \ldots, c_{m}$ to sets not equivalent under linear fractional transformation in the initial field $F$.

\author{
H. E. Slaught,
} Secretary of the Section. 